\title{
Philosophical-Mathematical Paradox of Dots
}

\author{
Hikmat N. Vazirov, Ph.D, \\ Fikrat H. Vazirov-Kangarli
}

\begin{abstract}
For the first time it was established, that geometric line constructed from dots is not solid, but a dashed line. And there can `t be solid lines without voids. The same words can be said about surfaces and planes. It is shown, that rational and irrational numbers are qualitatively different from a philosophical point of view and therefore not comparable. For this reason, they can't be placed on the same numerical axis. New mathematical-philosophical paradoxes had been considered. Today it is commonly supposed, that physical continuum is solid, continuous, without voids. It is shown, that continuum is having holes in truth. And the interaction of the holes of the continuum with its dots is the source of the philosophical movement. For the first time a new concept of philosophical freedom (archefolia) has been proposed in the article. To explain the essence of this new concept, the authors chose the methodology of mathematics.
\end{abstract}

Бог создал натуральные числа; всё остальное - дело рук человека. Л.Кронекер. Наука не сделает и шага вперед, пока философия не одобрит и не вдохновит ее на это. Т.Манн.

Хорошее начало полдела откачало. Поговорка. Великий оратор похож на заику. Дао де изин.

Наука не только должна находить и выяснять необходимые, общеполезные явления и законы мира (по-философски - вещи), но и пробуждать еще спящее сознание, предназначенное для поиска истины. И то обстоятельство, что среди исследователей мало глубоких и поэтому мало понимающих людей не повод заниматься только поверхностными проблемами и держать науку в дремоте. Поэтому нужно понять, что без правильных философских рассуждений и рефлексии найти истину будет невозможно. Но редко кто задумывается о том, что языков рассуждений может быть много, например, язык философии, язык математики, языки физики, права, экономики, формальной логики, бытовой язык и т.д. И ход, и результат рассуждений будет зависеть от языка. Так, например, в физике число $\boldsymbol{\pi}$ можно заменить на 3,14 , а вместо числа е можно писать 2,7. Действительно, если в измерениях получено 3,14 ампера, то никто не запрещает говорить $\boldsymbol{\pi}$ ампера. То же обычно можно делать и в математике, занимающейся количественными отношениями мира (реальности). Насколько это правильно? Только философия способна ответить на этот вопрос. Но сегодняшнее бездарное горе-руководство ВАК АР, упразднив философию для ученых, исследователей и докторантов, лишний раз доказало свою некомпетентность и неграмотность. Не поняв ценность, важность и необходимость философии для науки, она тем самым погубила будущее науки нашей страны. Ни один исследователь даже при всем желании не сможет избавиться от зависимости науки от философии. И при этом неприемлемые философские идеи, но не кажущиеся таковыми, обязательно приведут к неистинному и неполному пониманию и толкованию законов науки и самого мира. А это, в 
свою очередь, приведет к деградации, как самих исследователей, так и страны. Возможно, упразднить можно почти все, но только не философию! Самый великий гений всех времен Гегель - называл философию абсолютным знанием. И мы с удовольствием солидарны с ним и согласны с тем, что без философии нет деятельности вообще. Без философии нет ни науки, ни политики, ни дипломатии, ни права, ни религии, ни истории, ни этики, ни эстетики, ни искусства... одним словом, ничего. Философия - наиглавнейшее из всех человеческих дел. Недаром Гегель почти каждой из перечисленных областей человеческой деятельности посвятил произведение, например, «Философия права», «Философия религии» или, скажем, «Философия истории». Без философии нет и быть не может управления государством. Очень надеемся, что ВАК срочно исправит свою ошибку. Кодекс чести самураев «Бусидо» гласит: «Совершил ошибку и тут же исправил - считается, что ошибки не совершил». Остается только надеяться, что у ВАК совести все же немножко найдется.

Таким образом, становится понятным, что результат поиска истины зависит от способа рассуждения.

Итак, одной из известнейших проблем математики является древняя проблема происхождения и сущности чисел. Однако, эта проблема по сей день философски не решена. Причина в том, что с античных времен математика признавалась возвышенной наукой, не предназначенной для исследования природы, и поэтому на практике в экспериментах не использовалась. Поэтому ошибки и неправильности в самой системе и началах математики без экспериментов оказывались незаметными. Но эти неправильности приводили к неверным результатам уже впоследствии. И эти ошибки в системах и началах математики дают о себе знать и сегодня тоже. Поэтому Нобелевский лауреат Макс Лауэ в своей «Истории физики» пишет: «Из некоторых дошедших до нас высказываний Платона (427-347 до н.э.) мы узнаем о совершенном пренебрежении ко всякому эмпирическому исследованию. Любые попытки «осквернить» возвышенную науку - математику применением ее за пределами области чистых идей встречали резкое порицание. Именно с этим связано то, что Аристотель (384-322 до н.э.) в своей величественной системе науки дал в области естествознания логический или чаще только софистический анализ понятий, довольно некритически выведенных из поверхностно установленных фактов. Даже такой гений, как Архимед, не имел значительного влияния. Мы не знаем систематического исследования природы ни в древности, ни в средние века» [1]. И с развитием науки эти неправильности в системе математики и, в частности, в проблемах чисел, тоже развивались, расширялись и углублялись. И сегодня на данном этапе развития науки эта философская физико-математическая проблема приобрела новую актуальность, актуальность более высокого уровня [2]. Поэтому сегодня бросить новый взгляд на эту проблему - проблему чисел - является философской необходимостью.

Из элементарной математики известно, что числа удобно отображать на числовой прямой (оси). Выбрав две произвольные точки на этой прямой (удобно выбрать точку 0 и точку 1), устанавливают единицу длины, равную расстоянию между ними. Т.е. длина отрезка между точкой 0 и точкой 1 есть единица длины. И числа (точки), выбранные на этой числовой оси, будут расположены на каком-то расстоянии от точки 0. При этом это расстояние будет или 
соизмеримым с единицей длины, или несоизмеримым. Соизмеримым длинам отвечают рациональные числа. Это означает, что существует какой-то маленький отрезок на единице длины, из которого путем многократного повторения в точности могут быть получены и единица длины, и длина, соответствующая любому рациональному числу. Но, так получилось исторически, что на числовой оси расположили также такие числа, расстояния до которых никак не могут быть соизмеримы ни с единицей длины, ни с этим меленьким отрезком, каким бы маленьким он ни был. Такие числа называются иррациональными. Повторимся, расстояние от точки 0 до этого числа оказывается несоизмеримым ни с единицей длины, ни с этим маленьким отрезком. Частным случаем таких чисел являются трансцендентные числа (от лат. transcendere - переходить, превосходить). И размещение трансцендентных чисел на общей числовой оси приводит к любопытным парадоксам, на которые раньше внимания не обращали. Так, известно, что число $\boldsymbol{\pi}$ - трансцендентное число, т.е. это число не может быть корнем алгебраического уравнения, например, уравнения $\boldsymbol{x}^{2}=$ $\boldsymbol{\pi}$. Т.е. не существует такое число, квадрат которого в точности равен $\boldsymbol{\pi}$. На самом деле, $\boldsymbol{x}^{2} \neq$ $\boldsymbol{\pi}$. Да и само число $\boldsymbol{\pi}$ не имеет точного значения. Следует обратить внимание: точное значение $\boldsymbol{\pi}$ не существует! Т.е. речь не о том, что какое-то число в квадрате дает почти $\boldsymbol{\pi}$, т.е. приблизительно равно $\pi$, а том, что такого числа не существует в принципе. И нельзя утверждать, что это число есть $\sqrt{\pi}$. Это было бы тавтологией. Т.е., на самом деле, нет числа, квадрат которого точно равен $\boldsymbol{\pi}$ по той причине, что не может квадрат числа быть равным несуществующему. Но ведь существует функция $\boldsymbol{y}=\boldsymbol{x}^{2}$, считающаяся непрерывной. График этой функции - парабола. Но, в таком случае, на оси ординат на этом графике числу $\boldsymbol{\pi}$ соответствует какое-то число $\boldsymbol{x}$ на оси абсцисс. А этого быть не может, т.к. в таком случае это число $\boldsymbol{x}$ было бы тем числом, квадрат которого равен $\boldsymbol{\pi}$. Но такого числа, как сказано выше, просто не существует. А стало быть, в той точке параболы, ордината которой соответствует числу $\boldsymbol{\pi}$, должен быть разрыв (хоть и маленький, но все же разрыв). Т.е. парабола не является непрерывной линией?!. И таких разрывов на параболе должно быть бесконечно много, причем на любом участке графика, т.к. считается, что трансцендентных чисел бесконечно много на любом отрезке числовой оси. Стало быть, непрерывных линий в математике не существует вообще, даже в теоретических рассуждениях?!. Все они, в лучшем случае, квазинепрерывны, т.е. вроде бы непрерывны. Например, такой же разрыв будет и на той точке параболы, ордината которой соответствует числу е (основание натурального логарифма). Точно такие же разрывы будут наблюдаться, если рассмотрим уравнения $\boldsymbol{x}^{3}=\boldsymbol{\pi}$ или, скажем, $\boldsymbol{x}^{4}=\mathbf{e}$, а также бесконечное количество подобных функций с другими трансцендентными числами.

Или рассмотрим, например, задачу о квадратуре круга. Возьмем круг радиусом равным единице длины. Тогда площадь этого круга в современной общепринятой системе представлений будет $\boldsymbol{\pi}$ квадратных единиц. А квадрат площадью $\boldsymbol{\pi}$ будет иметь сторону длиной $\sqrt{\boldsymbol{\pi}}$. Т.е. задача о квадратуре круга сводится к построению отрезка длиной $\sqrt{\boldsymbol{\pi}}$ в масштабе любой принятой единичной длины. Но по общепринятой сегодня теореме о геометрических построениях, отрезок длины $\boldsymbol{\pi}$ построить невозможно. Т.е. на числовой оси 
отложить числа $\boldsymbol{\pi}$ и $\sqrt{\boldsymbol{\pi}}$ для любого масштаба, для любой принятой нами единицы длины, невозможно в принципе. Вот почему квадратура круга неосуществима.

Таким образом, проблема чисел и, в частности, проблема актуальной бесконечности, все же существует! И эта проблема весьма актуальна!

Однако привычными способами, устоявшимися в сегодняшней системе математики, ответить на возникшие вопросы и решить поставленные нами проблемы и парадоксы представляется уже невозможным, тем более что математику наукой признавать нельзя. Так, известные математики Э.Беккенбах и Р.Беллман начинают свою книгу «Введение в неравенства» словами: «Математику называют тавтологической наукой: другими словами, о математиках говорят, что они тратят время на доказательство того, что предметы равны самим себе. Это утверждение (свойственное философам) весьма неточно по двум причинам. Во-первых, математика, несмотря на свойственный ей научный язык, не является наукой; скорее ее можно назвать искусством, поскольку математическое творчество родственно художественному творчеству. Во-вторых, основные результаты математики чаще выражаются неравенствами, а не равенствами» [3]. Поэтому нужна правильная философия, являющаяся основанием и началом всех наук [4]. Недаром Евклид назвал свою книгу именно «Начала», потому что правильное начало важнее всего: хорошее начало - это уже половина всего дела. Так что же принято в современной системе науки и математики? Попробуем разобраться.

Первая книга «Начал» Евклида начинается словами: «1. Точка есть то, что не имеет частей. 2. Линия же - длина без ширины. Концы же линии - точки. Поверхность есть то, что имеет только длину и ширину» [5]. Смысл этих определений, возможно, заключается, в частности, в том, что и диаметр точки, и ширина линии, и толщина поверхности (плоскости) равны нулю. Кстати, из определения Евклида о том, что конец линии - точка, сразу возникает философский вопрос о том, а что находится на этой линии перед этой точкой. Правильный ответ такой: перед последней точкой находится предпоследняя точка. И сразу возникает вопрос: последняя и предпоследняя точки соприкасаются или нет? Но все равно, советские учебники по математике эти определения правильными не признавала. Более того, они утверждали, что ни точка, ни прямая определений не имеют вообще. А в советских учебниках по геометрии базовыми единицами являлись прямая и отрезок. Эти учебники давали следующие определения: «Отрезком называется часть прямой, которая состоит из всех точек этой прямой, лежащих между двумя данными ее точками. Эти точки называются концами отрезка. (Т.e., фактически считалось, что линия - это геометрическая фигура, состоящая из соприкасающихся точек - $\boldsymbol{A в m}$.). Окружностью называется фигура, которая состоит из всех точек плоскости, равноудаленных от данной точки. Эта точка называется центром окружности. Геометрическим местом точек называется фигура, которая состоит из всех точек плоскости, обладающих определенным свойством» [6]. Диаметр точки при этом, конечно же, предполагается равным нулю. Дадим наше определение линии и посмотрим, что из этого получится: «Линия - это целокупность точек, соприкасающихся то́лько с двумя соседними (кроме начальной и конечной)». Покажем это наглядно на рисунке 1. Из рисунка видно, что любая точка соприкасается только с двумя соседними. Исключение составляют 
лишь начальная и конечная точки, каждая из которых соприкасается только с одной точкой. Если уменьшить геометрические размеры (диаметры) этих «точек» (кружочков), оставив неизменными координаты их центров, то между «точками» окажутся пустоты (дырки). Если после этого «точки-кружочки» снова приблизить до их касания, а затем снова уменьшить их диаметры, снова получим пустоты между ними. И так до бесконечности. Наконец, в пределе, когда «диаметр» кружочка станет равным нулю (и кружочек превратится в настоящую геометрическую точку), то окажется, что эти, теперь уже настоящие точки, не смогут соприкасаться, а будут или сливаться друг с другом (одна точка ляжет на другую), или находиться на каком-то расстоянии друг от друга, не касаясь друг друга. Т.е. между любыми двумя точками окажется промежуток. А это значит, что понятие касания для геометрической точки с философской точки зрения исчезнет? Ведь касание означает, что одна часть вещи дотрагивается до какой-то части другой вещи, а другие части этой вещи - не дотрагиваются. Но ведь другой части у точки нет. Потому и касания нет.

Отсюда следует вывод: касательные, о которых говорилось в планиметрии - не совсем касательные, а возможно, лишь кажущиеся касательные, вернее, предполагаемые касательные, т.е. являются всего лишь конструктами, не имеющими референта. Не могут касаться друг друга вещи, не имеющие геометрического размера, вернее, если их размеры (диаметры) равны нулю. Они могут находиться или одна на другой, или находиться рядом друг с другом, не касаясь друг друга. А это значит, что математическое понятие непрерывности естественным и объективным образом исчезает, т.е. так же, как и понятие касательной, превращается в конструкт без референта. Получается, что непрерывной (сплошной) геоетрической линии нулевой ширины без разрывов (пустот, промежутков, дырок) быть не может вообще. Но даже если точки соприкасались бы, то, все равно, учитывая, что диаметр точки равен нулю, сколько бы соприкасающихся точек не ставить рядом, все равно, их суммарная длина будет равна нулю и линия не получится, т.к. $0+0+\ldots+0=0$.

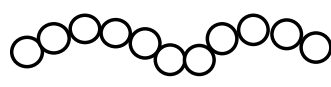

Рис. 1

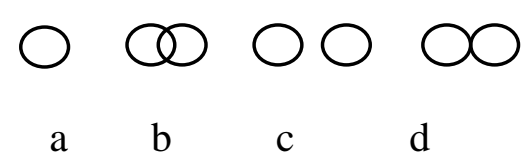

Рис. 2

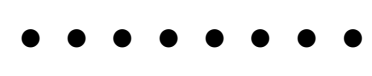

Рис. 3

Линия может получиться из точек и быть непрерывной лишь в случае, если она имеет ширину, т.е. если каждая «точка», из которой состоит эта «линия», имеет размер, больший нуля. Но, в таком случае, это уже будет не точка. А «линия» будет уже не линией, а геометрической фигурой, имеющая площадь, например, полоса, лента или круг (но не окружность). В случае же если ширина линии равна нулю, то значит, линия - прерывистая (т.е. содержит пустоты, дырки). В противном случае линия не получится. А если же «линия» все же име́ет ширину, то конец ее не может быть настоящей точкой, а будет кружочком, как на рис. 1. 
Здесь уместным будет сказать, что понятие «не имеет размера» с философской точки зрения не вполне корректно. С философской точки зрения все может иметь размер. Просто размер какой-то вещи или чего-то может быть равен нулю. Геометрическая точка и есть именно такая философская вещь, пространственный размер которой равен нулю. Т.е. точка безразмерна (имеет нулевую размерность). Поэтому, думается, Евклид ошибся, считая, что линия - это то, что не имеет ширины. На самом деле, вернее, на наш взгляд, с философской точки зрения, у линии ширина есть, но эта ширина равна нулю. Но, не имея никакого права критиковать этого великого гения, и ради истины и справедливости скажем, что Евклид и не говорил, что диаметр точки равен нулю. Он говорил только, что точка не имеет частей.

Аналогичными философско-математическими рассуждениями можем прийти к логическому умозаключению о невозможности сплошной плоскости. Любая плоскость, не обладающая толщиной (т.е. когда ее толщина равна нулю), должна быть равномерно дырявой. Сплошной (без дырок) может быть лишь «плоскость», обладающая толщиной, большей нуля. Т.е. любая плоскость (и геометрическая, и физическая) содержит бесконечное количество дырок, по количеству, возможно, равному количеству точек этой плоскости. Отсюда философский вывод: физическое пространство тоже дырявое...

Таким образом, как приведенные выше определения, так и наше определение линии неверны!?.

Итак, наши логические философско-математические рассуждения привели к умозаключению: плоские геометрические фигуры, например, окружность - прерывистые (дырявые), пунктирные «линии»! Такой же прерывистой (дырявой) является и любая геометрическая фигура, и любая линия. Это можно доказать логически, опираясь даже на один только логический закон исключенного третьего. Так, если считать (принять), что линия существует (причем не важно, объективно или лишь мысленно), то должно существовать лишь два возможных утверждения: 1) точки, из которых состоит эта линия, соприкасаются; и, 2) точки, из которых состоит эта линия, не соприкасаются. Из этих двух возможных утверждений только какое-то одно может быть истиной, а другое обязательно должно быть ложью. Если предположить, что истинно первое утверждение, а именно то, что точки соприкасаются, то учитывая то, что размер точки равен нулю, то, сколько бы соприкасающихся точек не ставить рядом линия не получится, т.к. $0+0+\ldots+0=0$. Но, мы же считаем (приняли), что линия существует. Значит, первое утверждение ложно. Отсюда выходит умозаключение: истинным является второе утверждение. Т.е. утверждение о том, что точки, из которых состоит линия, не соприкасаются - является истинным. Но тогда линия - прерывистая. Доказано!!!

Если же считать (принять), что линия не существует, то рухнет вся геометрия, которая на линиях и держится, и говорить не о чем. Но геометия же существует. Таким образом доказано: любые линии прерывистые, дырявые, пунктирные, т.е. являются отточиями (рис. 3 )...

Получается, что в евклидовой планиметрии вообще не может быть ни касательных, ни сплошных (без дырок) линий? Плоские геометрические фигуры или их части принципиально 
не могут касаться друг друга и быть сплошными, т.е. без дырок. Они могут лишь или лежать одна на другой (рис. 2, а), или пересекать одна другую (рис. 2, b), или же находиться на определенном расстоянии одна от другой (рис. 2, с). Касаться же, как на (рис. 2, d), они не могут.

И если рассмотреть ту самую числовую ось, то она тоже должна быть равномерно прерывистой (равномерно дырявой). И, учитывая то, что числовую ось придумали именно для чисел, известных на то время (т.е. для натуральных и дробных чисел, которые сегодня называют рациональными числами), и считали, что эти числа расположены на точках оси, мы должны думать, что те известные (рациональные) числа находятся именно на точках этой оси, а не в «дырках». А иррациональным «числам» на этой оси вообще-то места нет в принципе. Но до сих пор они туда были втиснуты. Это аналогично тому, что на ось, скажем, температур, втиснули время или расстояние. Но, если все же, иррациональные «числа» тоже силком загнать в эту ось (именно это положение в системе сегодняшней математики и существует), то придется предположить, что иррациональные «числа», частным случаем которых являются трансцендентные «числа» (например, $\boldsymbol{\pi}, \mathbf{e}, \boldsymbol{\alpha}^{\mathbf{n}}$ для некоторых $\alpha$ и n), расположены как раз в дырках этой прямой?

Итак, получился неизвестный ранее философско-математический парадокс точек, который можно сформулировать, например, так: сплошная линия существует, и она состоит из соприкасающихся точек, но сплошная линия не существует, т.к. точки касаться друг друга не могут, и поэтому это не линия, а что-то вроде пунктира, т.е. нечто разорванное (с дырками). Этот парадокс можно сформулировать иначе: линия образу́ется (состоит) из соприкасающихся точек, но линия не́ образуется, т.к. точки соприкасаться не могут, но даже если точки соприкасались бы, то линия все равно не образовалась бы, т.к. диаметр точки равен нулю и их сумма была бы равна нулю. Можно и так: точки соприкасаются, и поэтому образуется линия, но они не соприкасаются, т.к. соприкасаться не могут, и поэтому линия не образуется. И разрешить этот парадокс или апорему, представляется достаточно сложным.

Здесь как раз уместным будет дать объяснение понятию иррационального «числа». Иррациональное «число» - это «число», которое не может быть получено делением одного натурального числа р на другое натуральное число q. Например, квадратный корень из числа 2 является иррациональным «числом» (т.к. никак не может быть получен делением одного натурального числа $\mathbf{p}$ на другое натуральное число $\mathbf{q}$ ), и является бесконечной непериодической десятичной дробью. Но это «число» явля́ется корнем алгебраического уравнения $\mathrm{x}^{2}=2$. В то же время, имеются такие иррациональные «числа», которые корнем алгебраического уравнения являться не могут. Такие иррациональные «числа» называются трансцендентными «числами». Таковыми являются, например, числа $\boldsymbol{\pi}$, е, десятичные логарифмы всех целых чисел, не кратных 10, или числа вида $\mathbf{a}^{\mathbf{b}}$, где, $\mathbf{a}-$ алгебраическое число, не равное 0 и 1 ( $\mathbf{a} \neq 0 ; \mathbf{a} \neq 1)$, a $\mathbf{b}-$ иррациональное алгебраическое число. Есть бесконечно много и других трансцендентных чисел.

Становится понятным, что любые две философские вещи, имеющие пространственный размер, могут находиться (располагаться) по отношению друг к другу только четырьмя 
способами, как показано на рис. 2: а) конгруэнтно, т.е. одна целиком лежит на другой; b) пересекаться, т.е. одна частично лежит на другой; с) одна находится на каком-то расстоянии от другой, не касаясь ее. Положение же, изображенное на рис. $2 \mathrm{~d}$ - всего лишь абстракция. Но ведь у точек (материальных, физических, геометрических, ..., и вообще, любых точек) пространственные размеры равны нулю (т.е. их диаметры равны нулю). Поэтому они касаться друг друга не могут, а могут быть только в двух положениях: либо одна точка полностью лежит на другой, (рис. 2 а), либо между ними имеется какое-то расстояние (рис. 2 c). Дело в том, что, повторимся, касание одним телом другого означает соприкосновение, «дотрагивание» наименьшей части первого тела наименьшей части второго тела. Наименьшая же часть - это точка, а у точки частей нет по определению. Поэтому точки соприкасаться не могут. Принципиально. И вообще, не ограничивая общности, вещи (в философском смысле), не имеющие частей (пространственный размер равен нулю в любом направлении), соприкасаться не могут. Они или лежат одна на другой, или же находятся на каком-то расстоянии друг от друга. Явление соприкасания для вещей, размер которых равен нулю, с философской точки зрения становится дистинктивно невозможным. Или, говоря философским языком, две точки не могут иметь непосредственного отношения друг к другу. Но они имеют отношение к рядом стоящим дыркам. Но по правилам отношения транзитивности: если $\mathrm{xRy}$ и $\mathrm{yRz}$, то $\mathrm{xRz}$, где $\mathrm{R}$ - символ философского или логического отношения. Поэтому, если точка имеет отношение к ближайшей дырке, а эта дырка к следующей точке, то первая точка имеет отношение к той второй точке через дырку между ними. Получился еще один философско-математический парадокс: точка к ближайшей точке отношения не имеет, но точка к ближайшей точке отношение име́ет (через дырку). Так через дырку и передается отношение и движение.

И, если все же силой загнать (втиснуть) иррациональные «числа» в ось для рациональных чисел (как уже сказано выше, именно такое положение в сегодняшней системе математики имеет место), то из сказанного выше выйдет гипотетическое философско-математическое умозаключение: количество иррациональных «чисел» (мощность или число элементов системы иррациональных «чисел»), уж по крайней мере, не меньше количества рациональных чисел. При этом если рациональные числа находятся на точках той известной условной числовой оси, то иррациональные числа должны быть между точками (в дырках) этой оси (хотя вообще-то им на этой оси места нет - для них нужна другая ось).

Таким образом, для неправильной, но сегодня принятой, системы одной единственной числовой оси получилась философско-математическая теорема: между любыми двумя рациональными числами на общепринятой сегодня числовой оси находится хотя бы одно иррациональное число и, в частности, трансцендентное «число». А также возникает гипотеза: эти два вида чисел - рациональные и иррациональные - распределены на принятой числовой оси неравномерно, неравноплотно, неравномощно или неравноколичественно!?. Почему? Постараемся логически обосновать эту гипотезу (не хотим называть это теоремой). Мы уже объяснили, что, если пространственный размер точки равен нулю, т.е., говоря принятым сегодня языком, размера нет, тогда эти точки соприкасаться друг с другом не смогут, и поэтому линия не получится. Но даже если они соприкасались бы, то линия все равно не 
образовалась бы, т.к. диаметр точки равен нулю, а сумма нулей равна нулю. Поэтому, для того, чтобы образовалась линия из точек, необходимо считать, что между точками имеются промежутки, дырки. И тут возникает еще один философско-математический парадокс: минимальный размер промежутков между точками должен быть равен размеру точки, но размер промежутков между точками не может быть равен размеру точки, т.к. иначе линия не получится. Этот парадокс возникает потому, что если пространственный размер промежутков между точками равен размеру точки, т.е. равен нулю, то это фактически означает, что этих промежутков нет. Но, как показано выше, промежутки есть. Поэтому, чтобы разрешить этот парадокс, мы должны считать, что размер промежутков (мы их еще назвали по аналогии с физикой полупроводников - дырками) больше нуля, т.е. больше размера точек, а стало быть, в каждую из этих дырок можно втиснуть столько «чисел», что их количество будет больше единицы? При этом на каждой точке числовой оси находится только одно число, и это число - рациональное число. Тогда, в случае, если мы вынуждены будем разместить иррациональные «числа» тоже на этой же прямой (числовой оси), то придется их разместить в дырках этой прямой, хотя этим «числам» там места вообще-то нет. Причем, получается, что количество иррациональных чисел больше (или, по крайней мере, не меньше), чем рациональных чисел, т.к. дырка по размеру больше точки и, поэтому, там может расположиться большее количество «чисел». Если число всех возможных трансцендентных чисел на каком-то отрезке числовой оси бесконечно, тогда пустых (без трансцендентных чисел) дырок, наверное, быть не может. Если же число всех существующих трансцендентных «чисел» конечно, то между точками мо́гут оказаться пустые дырки (без трансцендентных «чисел»). Итак, каждой точке соответствует только лишь одно рациональное число, а каждой дырке, расположенной между двумя стоящими рядом точками, наверное, соответствует бо́льшее количество неоправданно и необоснованно втиснутых туда иррациональных «чисел». Во всяком случае, в каждой дырке количество иррациональных «чисел» больше единицы. А это означает, что если искусственно, т.е. силой, загнать иррациональные «числа» в ось для рациональных чисел, то количество всех иррациональных «чисел» на любом отрезке общепринятой числовой прямой, окажется больше количества всех рациональных чисел. Т.е. получается, что вообще-то количество всех возможных иррациональных «чисел» больше количества всех возможных рациональных чисел. Этот результат, полученный философскими методами, не будет трудной теоремой для математиков.

Предлагаемая нами философская логико-математическая теория, очевидно, применима в любой математике и геометрии, например, геометрии Лобачевского или геометрии Римана.

Итак, повторимся, если точка имеет пространственный размер, равный нулю (т.е. не имеет частей), то, сколько бы точек не ставить рядом - линия не получится. Действительно: $0+0+\ldots+0=0$, и длина будет равна нулю. Поэтому, для того, чтобы из точек все же построить линию, необходимо, чтобы или пространственный размер точки был равен не нулю, а был равен бесконечно малой величине, но большей нуля (но, в этом случае, концы линии не будут точками, а будут кругами или шариками). Или же, если размер точки равен нулю, тогда между точками должны быть 
расположены дырки, т.е. оставаться пустоты, промежутки, т.к. в противном случае линия не образуется. Т.е. реальная линия теперь должна выглядеть как на рис. 3.

Таким образом, вопреки устоявшемуся мнению о сплошных линиях, на самом деле, линия, например, числовая прямая - прерывистая, дырявая конструкция, т.е. напоминает пунктир, а точнее, отточие (рис. 3).

В повседневной жизни используется большое количество чисел. И этого количества действительных (рациональных) чисел полностью хватает, например, для проведения какихто экспериментальных исследований. Но, большие проблемы возникают сегодня, когда речь заходит о философских или теоретических рассуждениях. Например, если нужно точно (именно точно) установить длину $\mathbf{d}$ диагонали квадрата со стороной $\mathbf{b}$, то получим, что $\mathbf{d}=$

$\sqrt{2} \mathbf{b}$. И при этом выяснится, что $\sqrt{2}$ - это и не целое число, и не дробное. На момент открытия, это «число» было «новым видом чисел», и назвали такие «числа» иррациональными числами. Повторимся, отличие их от рациональных чисел, т.е. целых и дробных чисел, в том, что последние можно выразить в виде отношения двух целых чисел, например, $\mathbf{p}$ и q, поэтому их и называют рациональными числами, т.е. полученных делением, отношением. Иррациональные же «числа» потому и называются иррациональными (что в переводе означает «не имеющие отношения»), что их точно получить отношением двух целых чисел р и q невозможно. Сама группа иррациональных «чисел» делится на две части: одна часть - это алгебраические «числа», которые мо́гут быть корнями алгебраического уравнения с целыми коэффициентами, другая часть состоит из «чисел», которые не́ могут быть корнями алгебраического уравнения. Те иррациональные «числа», которые не́ могут быть корнями алгебраического уравнения, называются трансцендентными «числами». Примеры трансцендентных «чисел» приведены выше.

Таким образом, рациональные и иррациональные числа в философском смысле являются разнородными, разнотипными, противоположными, поэтому для их геометрического изображения требуются разные числовые оси. Т.е. эти два вида чисел различны качественно: рациональные числа гомогенны, иррациональные - гетерогенны. Их неодинаковость проявляется в каком-то сходном свойстве, но внутренне, онтологически эти числа друг с другом не связаны. Думается, что противоположность этих чисел как внешняя, так и внутренняя. Эти числа взаимно отрицают, исключают друг друга. Такими же философски противоположными, качественно разными, разнородными, разнотипными, отрицающими друг друга, являются алгебраические и неалгебраические числа, действительные и комплексные числа...

Если бы философски можно было допустить, что числа как вещи сделаны из чего-то, то рациональные числа гомогенны (однородны), т.е. «сделаны» из одного и того же. Поэтому любое из этих чисел может быть получено из других таких же чисел. И поэтому они сравнимые, т.е. одно больше или меньше другого, или равно другому. Комплексные числа негомогенны, они гетерогенны, т.е. «сделаны» из не одного и того же или же не из одного и того же. Поэтому эти «числа» несравнимые. Т.е. нельзя спрашивать, какое из двух комплексных «чисел» больше, а какое - меньше. Ни одно из них и не больше, и не меньше другого. Они несравнимые, т.к. разнородные, качественно разные. Потому и разместились 
они (совершенно неосознанно) не на одной оси, а в разных местах плоскости, образованной двумя разными осями. То же можно сказать и об иррациональных «числах». Ни одно из иррациональных «чисел», в частности, трансцендентных «чисел», не может быть получено из других трансцендентных чисел, если, конечно, не прибегнуть к особым фиктивным, химерным ухищрениям. Кроме всего, принято считать, что действительные, вещественные числа являются частным случаем комплексных «чисел». Тут все зависит от договоренности (научно-философская теория конвенционализма). Если мы договоримся признавать комплексные «числа» чи́слами, тогда действительные числа окажутся частным случаем комплексных «чисел», как и пишут в математических справочниках. Однако ведь действительные числа выражают количество, а сами же эти справочники утверждают, что комплексные «числа», наоборот, количества не выражают! Значит, комплексные «числа» в этом отношении не должны признаваться числами, и, в этом случае, действительные числа не могут признаваться частным случаем комплексных «чисел». Тогда получается, что общее (индуктивное) не выполняет то, что выполняет частное. Философски это невозможно. Таким образом, именно с философской точки зрения, действительные (вещественные) числа не должны признаваться частным случаем комплексных «чисел». Действительно, философским качеством действительных чисел является свойство (физическое качество) выражать количество. А комплексные «числа» не обладают свойством или качеством выражать количество (это утверждают все учебники по математике). Таким образом, необходимо признать, что комплексные «числа» и действительные числа - это разнокачественные в философском отношении вещи. Именно поэтому комплексные числа размещают совершенно неосознанно не на линии, а на плоскости, образованной двумя неравноценными ортогональными числовыми осями - осями действительных и мнимых чисел. Кроме этого, повторимся, каждое рациональное число имеет референта, а комплексное «число» - референта не имеет. Трансцендентные «числа» также референта не имеют. И то, что из одного комплексного «числа» можно вычитать другое комплексное «число» - является философским недоразумением (но, не математическим). Но это недоразумение в сегодняшней системе науки является нормой, и это можно увидеть в любом учебнике.

Возможно, и нуль числом признавать не следует - это всего лишь условное обозначение (знак, символ) отсутствия. Т.е. нуль - это пустое понятие, которое не отображает никаких объектов так же, как, например, понятие «хорда треугольника» или, скажем, «периметр электрона». Т.е. это пустое понятие, пустой класс, т.е. класс чего-то, который не содержит в себе никаких элементов. Например, класс «квадратных треугольников» является пустым классом, т.к. квадратных треугольников не бывает так же, как и треугольных квадратов, хорды треугольника или периметра электрона. И нельзя путать «часть целого» с «частным случаем». Часть целого (например, подшипник трактора) может делать то, что не может целое, а целое (например, трактор) может делать то, что не может часть целого (подшипник). Частный случай же не может совершать то, что не может общее. А общее может то, что может частное, и может то, что не может частное. 
Этими рассуждениями, мы хотели показать, что рациональные и иррациональные числа с философской точки зрения являются разнородными, разнотипными, качественно разными вещами. А их всех в свое время под названием «действительные числа» совершенно необоснованно разместили на одной общей числовой оси. И эта неправильность сегодня продолжается. Т.е. под одним термином «действительные числа» до сих пор некорректно называют на самом деле разнокачественные, взаимоисключающие вещи. Качественное отличие всех тех чисел, которые неосознанно и неудачно подстригли под одну гребенку «действительных чисел», заключается также в том, что часть этих чисел, а именно, рациональные числа, имеют референта. Иррациональные же числа, в том числе, трансцендентные числа, референта не имеют. Они являются только лишь концептами или конструктами. Поэтому в предлагаемой новой системе науки рациональные числа нельзя сравнивать с иррациональными. Будучи разнокачественными вещами в философском смысле, они не подлежат сравнению. Так, нельзя сравнивать, что лучше, телевизор или диван, т.к. они - вещи разнокачественные и поэтому несравнимые. Точно так же нельзя сравнивать арбуз с телефоном, т.к. они вещи разнокачественные. Разнокачественные вещи друг с другом сравнивать нельзя. Точно так же, вещи, имеющие референта, отличаются от вещей, не имеющих референта, качественно, и поэтому не могут быть сравнимыми!

Отсюда получается невероятное умозаключение: $\pi$ (которое сегодня считается равным приблизительно 3,14 ) не меньше 4 и не больше 3 , потому что $\pi$ нельзя сравнивать ни с 3 , ни с 4 , т.к. 3 и 4 являются натуральными числами, а $\pi$ трансцендентное «число»? Т.е. с философской точки зрения это разнородные, разнокачественные вещи. И этот результат тоже парадокс: считается, что $\boldsymbol{\pi}$ равно примерно 3,14 и поэтому $\boldsymbol{\pi}$ больше 3 и меньше 4. Но $\boldsymbol{\pi}$ не равно 3,14 и поэтому его нельзя сравнивать ни с 3 , ни с 4, потому что $\pi$ и эти два числа разнокачественные вещи. Значит, «число» $\boldsymbol{\pi}$ с философской точки зрения и не больше 3 , и не меньше 4? И утверждение, что « меньше 4, но больше 3» такое же неверное, как и утверждение, что 3,14 метра меньше 4 килограммов, но больше 3 секунд (ни метры, ни секунды нельзя же сравнивать с килограммами: в философском смысле это разнокачественные вещи)? Трансцендентные числа референта не имеют, и, поэтому, они отличаются от рациональных чисел качественно, т.е. это разнокачественные вещи. И попытка сравнить рациональные числа с иррациональными числами также напоминает попытку, например, сравнить человека с его фотографией или телевизором. Это очень важно, поэтому еще раз повторимся. Нельзя сравнивать длину, скажем, с массой, или площадь с силой электрического тока. Это неравнокачественные, разнокачественные вещи. Поэтому нельзя спрашивать, что больше, 5 метров или 20 килограммов. Или, что меньше, 2 кубических метра или 2 ампера. Все они обозначают количественные характеристики разных свойств философской вещи. И поэтому их нельзя сравнивать. Нужно понять, что $\boldsymbol{\pi}$ не является числом не потому, что его не смогли точно измерить, а потому, что оно не может быть точно измерено, т.к. этого точного значения просто не существует, а стало быть и числа такого не существует. Поэтому $\boldsymbol{\pi}$ не выражает количество, а рациональные числа выражают точное количество, и поэтому рациональные 
числа действительно являются числами. Точно так же, выражение $\lg 12=\mathbf{A}$ и выражение

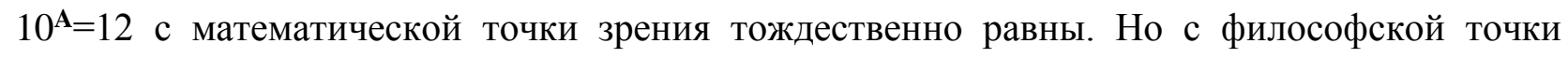
зрения, такие выражения являются фикцией, т.к. нет такого числа А, для которого данные выражения выполнялись бы точно. Такое число А просто не существует. Т.е. с философской точки зрения $10^{\mathbf{A}}=12$ ни для каких $\mathbf{A}$ не выполнимо точно. Но с математической или физической точек зрения это выражение возможно, и А при этом является трансцендентным «числом» «несколько большим единицы». Т.е. с философской точки зрения, на числовой оси для такого «числа» А места нет вообще́, потому, что такое «число» для философа не существует. Но для математика или физика, это «число» существует, и если неоправданно все же расположить это «число» на общепринятой числовой оси, то придется расположить его между 1 и 2.

Продолжая философские рассуждения о сплошных (несплошных) линиях, придем к выводу, что не бывает сплошных плоскостей (т.е. плоскостей без дырок), сплошного объема или сплошного пространства без дырок. Т.е., на самом деле, физический вакуум дырявый, как и плоскость, как и прямая или любая другая линия. И в «дырках» числовой плоскости, наверное, находятся иррационально-комплексные или трансцендентнокомплексные «числа»).

Для дальнейшего разъяснения нашей философско-математической теории или новой системы науки нам понадобится понятие континуума. В математике для геометрической интерпретации действительных чисел континуум представляли точками числовой прямой. Каждая точка этой прямой соответствовала какому-либо действительному числу. Теперь же стало понятно, что если сегодня продолжать принимать за истину правильность числовой оси, то не только каждая точка, но также и дырки между точками будут соответствовать действительным числам (в дырках будут находиться иррациональные числа, которые сегодня пока все еще совершенно необоснованно тоже признаются действительными числами). Но, как показано выше, это не́ правильно. Поэтому для каждого вида чисел необходимо вводить отдельные числовые оси: для рациональных чисел можно использовать ту ось, которую сегодня называют числовой прямой (но, как удалось установить, она дырявая). Для иррациональных «чисел», которые качественно отличаются от рациональных чисел, необходимо вводить свою ось, т.к. им на точках оси рациональных чисел места нет, и в эту ось их неосознанно и необоснованно загнали силой, причем совершенно неоправданно. Поэтому, если уж загонять иррациональные числа в ось рациональных чисел, то придется расположить их в дырках этой оси. Этими рассуждениями мы объяснили неправильность старой системы математики и теории чисел. Даже для отрицательных «чисел» необходимо вводить отдельную ось.

В физике под континуумом понимают пространство-время. Структура физического континуума зависит от плотности вещества, излучения, поля. Физический континуум должен быть охарактеризован или непрерывностью, или прерывностью. Сегодня общепринято, что физический континуум непрерывен, т.е. он сплошной, без пустот, без дырок.

\section{Вопреки общепринятому сегодня, мы утверждаем, что и математический} континуум, и физический континуум, являются прерывистыми, дискретными, т.е. 
содержат пустоты, дырки. И число пустот или дырок хотя и равно числу составных частей матрицы континуума (т.е. точек), но пространственный размер дырок должен быть больше нуля. Если это физический континуум, то материалом матрицы (назовем этот материал основой) является вакуум. Если это математический континуум, то матрицей, т.е. «материалом» континуума, являются числа.

Наши логико-философско-математические рассуждения необходимы были для того, чтобы показать, что точки и дырки, о которых мы говорим - это сущность основы философского движения вообще и движения микрочастиц, в частности. И в частности флюктуаций вакуума. Конечно же, трудно себе представить это, но именно наличие в физическом континууме точек (и дырок между ними), и взаимодействие их между собой через дырки и есть источник философского движения и бытия - свобода (архефолия). Вернее, не наличие, а то, что сам физический континуум состоит из точек и дырок, и является источником движения всего сущего, бытия. И взаимодействие точек с дырками мы назвали архефолией $[<$ гр. arche начало, лат. folleo - ходить взад вперед, болтаться и порт. folia - безумная пляска, шумное веселье].

Гегель считал, что противоречие является, во-первых, основанием любого движения, вовторых, сущностью вещей. Т.е. между точкой и дыркой (являющейся местом отсутствия субстанции-материи-вещества-континуума-вакуума-бытия) возникает диалектическое противопоставление, противоположение, противоречие и взаимодействие. Мы считаем, что противоречие обладает одним очень важным свойством, заключающимся в том, что противоречие между, скажем, двумя вещами, возможно только в том случае, когда одна вещь содержит в себе часть или хотя бы элемент второй вещи. В противном случае противоречие возникнуть не может. Т.е. противоречие может быть только в системе. Так, например, между морем и трактором, стоящим далеко от моря, нет взаимодействия и поэтому противоречия тоже нет. Если же трактор будет находиться в море (в воде), то образуется система моретрактор, и возникает взаимодействие и противоречие, приводящее к коррозии и разрушению трактора. И в сущности противоречия философской вещи кроме двух составных частей (противоположности и взаимопроникновения) должна содержаться третья составная часть, которую мы назвали архефолией. Вернее, архефолия - это сущность противоречия. И именно благодаря этой архефолии и осуществляется взаимодействие противоположностей, т.е. противоречие, приводящее к его разрешению (снятию). Т.е. все сущее дырявое. И, возможно, все могло бы быть в состоянии полного покоя. Но, если что-то приведет к взаимодействию даже всего лишь одной точки с одной дыркой, то это приведет к передаче взаимодействия от одной точки или одной дырки к другим, а затем и всем точкам, и дыркам, а это в свою очередь к вечному движению. Взаимодействие дырок континуума с его точками - архефолия - и есть основа философского движения. Уместным будет сказать, что отличительной особенностью именно диалектического противоречия является то, что, в отличие от формально-логического противоречия, архефолия проявляется именно в предметном противоречии, т.е. в референтах. В конструктах же архефолии, наверное, нет! И, без учета архефолии, опосредствующей противоположности в противоречии, решать диалектические задачи философского движения и развития, а также науки, не удастся. 
Таким же прерывистым является и время. И взаимодействие дырки со своим окружением, т.е. с другими дырками и точками - это та причина, которая приводит и к известному в физике принципу неопределенности, и, наверное, к возможному отделению пространства от времени.

Авторы настоящей статьи, получившие изложенные результаты логико-философскими рассуждениями, уверены, что в ближайшее время физики докажут эти результаты экспериментально. Можно предсказать также, что к известным сегодня четырем силам (взаимодействиям) в природе, а именно, гравитационным, электромагнитным, сильным и слабым, может быть добавлена пятая сила. Эта сила отвечает за так называемую темную материю. Находится темная материя, по всей видимости, в предложенных нами дырках физического континуума. И энергии в этих дырках физического пространственновременного континуума намного больше известной нам сегодня энергии Вселенной. И пространственно-временной континуум можно назвать континуумом бытия-небытия.

В заключение, дабы не обижать самолюбие «беотийцев» шокирующими их сознание результатами настоящего сообщения, приведем небольшой отрывочек из И.Пригожина. «В своих «Темах» Мерло-Понти утверждал, что «философские» открытия естествознания, концептуальные преобразования его основ нередко происходят в результате негативных открытий, служащих толчком к пересмотру сложившихся взглядов и отправным пунктом для перехода к противоположной точке зрения. Доказательства невозможности или несуществования (будь то в теории относительности, квантовой механике или термодинамике), показали, что природу невозможно описать «извне», с позиций зрителя. Описание природы - живой диалог, коммуникация, и она подчинена ограничениям, свидетельствующим о том, что мы - макроскопические существа, погруженные в реальный физический мир» [8].

\section{В Ы В О Д Ы}

1. Вещи, не имеющие пространственного, геометрического размера, т.е. если их пространственный размер равен нулю, друг друга касаться не могут дистинктивно. Это значит, что понятие непрерывности исчезает, превращаясь в конструкт или концепт без референта, и если считать, что линии существуют, то непрерывных линий без разрывов быть не может. То же можно сказать и о плоскостях: если считать, что плоскость существует, то любая плоскость, у которой толщина равна нулю, должна быть равномерно дырявой. Пространство тоже дырявое, т.е. прерывистое, а не непрерывное. Поэтому, базовыми единицами в нашей теории являются: 1) точка (как и у Евклида); 2) дырка; 3) отточие (у Евклида - прямая); 4) сетка (у Евклида - плоскость).

2. Первый парадокс точек: считается, что сплошная (гладкая, непрерывная) линия существует, и она состоит из соприкасающихся точек, но сплошная линия не существует, т.к. это не сплошная непрерывная линия, а что-то вроде пунктира, т.е. нечто разорванное и с дырками. Т.е. считалось, что точки соприкасались, и поэтому образовывалась линия. Но они соприкасаться не могут, и поэтому сплошная линия образоваться не может. 
3. Второй парадокс точек: на числовой оси или на любой линии геометрический размер промежутков между безразмерными точками должен быть равен размеру точки, но размер промежутков не может быть равен размеру точки.

4. Третий парадокс точек: до сих пор считалось, что $\pi$ больше 3 , но меньше 4. Но мы установили, что $\pi$ нельзя сравнивать ни с 3 , ни с 4, потому, что это разнородные, разнокачественные вещи, и поэтому $\pi$ и не больше 3 , и не меньше 4, и не меньше 3 , и не больше 4, и не равно 3, и не равно 4. Но в математике «не больше» - это то же, что и «меньше или равно», т.е. $\leq$, а «не меньше» - это то же, что и «больше или равно», т.е. $\geq$. Поэтому можно написать, что $3 \geq \boldsymbol{\pi} \geq 4$ ? Таким образом, с одной стороны принято, что, $\boldsymbol{\pi}$ больше 3 и меньше 4, и потому можно написать $3<\pi<4$, но, с другой стороны, получается, что $3 \geq \boldsymbol{\pi} \geq 4$ ? Можно также показать, что $\boldsymbol{\pi}=3$ и $\boldsymbol{\pi}=4$. Такие противоречия получились изза того, что мы попытались сравнивать несравнимые вещи, а именно трансцендентное «число» $\boldsymbol{\pi}$ с нетрансцендентными (натуральными) числами 3 и 4, что и привело к этому парадоксу. Для несравнимых вещей знаки сравнения больше $(>)$, меньше $(<)$, не больше $(\leq)$ или не меньше $(\geq)$ не применимы и не имеют смысла, т.к. несравнимые вещи сравнивать нельзя. Таким образом, установлено, и поэтому необходимо понять, что количественный критерий натурального ряда не применим к трансцендентным числам. Т.е. свойства бытия нельзя применять к небытию. И вообще, думаем, бытие нельзя сравнивать с небытием. Правда, хотя великий Гегель и утверждал, что бытие и небытие - это одно и то же [9], но думаем, в нашем случае это не так. Поэтому $\boldsymbol{\pi}$ нельзя сравнивать ни с 3 , ни с 4 , ни с другими натуральными числами. Натуральные и вообще, рациональные числа - это бытие, а трансцендентные числа - это небытие. Т.е. для $\boldsymbol{\pi}$ обычно берут значение 3,14 , но необходимо понять, что $\boldsymbol{\pi}$ - это не 3,14 и даже не число приблизительно равное 3,14 , а совсем другая вещь в философском смысле. Т.е. трансцендентные «числа» - такая же химера, как и квадратный корень из арбуза, логарифм треугольника или компас в четвертой степени. Т.е. трансцендентное «число» - это условное обозначение чего-то несуществующего, вернее феноменологического. И для $\boldsymbol{\pi}$ места на принятой числовой прямой нет. Но если $\boldsymbol{\pi}$ силком загнать в принятую сегодня числовую ось, то это «число» окажется в дырке. Возможно, прав был Л.Кронекер, утверждая, что бог создал натуральные числа; все остальное - дело рук человека.

Еще раз объясним популярно, почему так получается. Дело в том, что обычно у референтов бывает много предикатов, свойств и параметров. Так, если взять телевизор и ведро, то увидим, что и у телевизора, и у ведра есть много схожих параметров, например, масса, площадь поверхности, объем, цвет и др. Поэтому их можно сравнивать по какому-то отдельному параметру, например, массе. Т.е., несмотря на то, что телевизор и ведро разнокачественные вещи в философском смысле, все равно, их можно сравнивать по какомуто одному свойству, присущему им обоим. В случае чисел это не так. Дело в том, что у чисел есть только одно свойство - это свойство выражать количество. Но, как было показано выше, многие из так называемых чисел на самом деле числами не являются, т.е., например, натуральные числа и иррациональные «числа» разнокачественные вещи. И учитывая, что у чисел вообще-то есть только одно свойство - выражать количество, и этим свойством 
обладают только рациональные числа, а иррациональные «числа» и, в частности, трансцендентные «числа», таким свойством, в общем-то, не обладают, то эти числа нельзя сравнивать. Т.е. трансцендентные числа референтом не обладают. И $\boldsymbol{\pi}$ такая же химера, как и черт, но мы же слово черт используем, хотя такого референта нет. Так же и л. Поэтому, рациональные числа, например, 3 и 4, совершенно отличаются от «числа» $\pi$, т.е. это разнокачественные вещи. И учитывая то, что у любых чисел других свойств, кроме как выражать количество, нет, приходим к выводу, что их сравнивать нельзя. Поэтому и получилось, что $\pi$ и не больше 3, и не меньше 4 (!?).

5. Четвертый парадокс точек: до сих пор думали, что поверхность и, в частности, плоскость, существует. Но плоскость не существует, т.к. это не поверхность, а нечто дырявое, т.е. что-то вроде сетки. Т.е. поверхность - это нечто дырявое, вроде дуршлага.

6. Между любыми двумя рациональными числами на общепринятой сегодня (но неправильной) числовой оси, находится хотя бы одно иррациональное «число», которое туда втиснуто необоснованно. Это необоснованное расположение иррациональных чисел на оси рациональных чисел аналогично тому, что на оси скоростей расположили массу или напряжение. Т.е. для каждой физической величины требуется (и поэтому необходимо) ввести отдельную ось.

7. Рациональные и иррациональные числа в философском смысле (не математическом!) являются противоположными, и эти два вида чисел различны качественно, и поэтому они несравнимы. Для них требуются две различные числовые оси.

8. Минимальная в пространственном отношении (по размеру) вещь в мире - или точка континуума, или его дырка. Т.е. существует квант длины. А дырка - это место, свободное от точки, т.е. «материала» континуума.

9. Длина окружности несоразмерна с ее диаметром потому, что и окружность, и диаметр (отрезок) - дырявые. Если увеличить длину окружности на одну дырку - его диаметр увеличится на один квант длины, и все равно, они останутся несоразмерными (несоизмеримыми). И если окружность разрезать в одном месте и выпрямить, то если до сих пор думали, что длина получившегося отрезка окажется равной длине этой окружности, то на самом деле, длина получившегося отрезка окажется меньше длины той окружности на один квант длины. И если разделить длину этого получившегося отрезка на диаметр той окружности, то трансцендентность исчезнет!? И если увеличить длину окружности на один квант длины, то диаметр увеличиться не сможет!?

10. Физический континуум, введенный по аналогии с математическим континуумом, и изначально считавшийся континуальным, т.е. непрерывным (сплошным), на самом деле является прерывистым, т.е. с дырками. Взаимодействие дырок физического континуума с его точками (т.е. архефолия или взаимодействие бытия и небытия) есть и начало, и основа, и основание философского движения.

11. Установленный недавно физиками теоретический феномен кротовой норы, через которую возможно перемещение из одной галактики в другую, по всей видимости, может осуществляться именно через дырки физического континуума, т.е. через небытие, причем со скоростью намного превышающей скорость света в вакууме. И так называемые 
элементарные частицы, например, протоны, нейтроны - тоже дырявые (пористые). Пространство тоже дырявое (пористое). Если предположить, что Большой взрыв действительно имел место, то он потому и произошел, что та первичная точка была пористая, и именно взаимодействие ее дырок с ее точками и привело к взрыву. Кстати, Большой взрыв, возможно, произошел не в одной точке, а в нескольких, и это был разрыв вакуума. Возможно вся масса Вселенной не была в одной точке, а возможно, этой точки не было вообще, т.к. если все образовалось из точки, то вокруг этой точки должно было быть гравитационное поле, а это значит, что было пространство, где это поле было. А это противоречит всей теории Большого взрыва. Таким образом, можно считать, что к движению (взрыву) привело взаимодействие бытия с небытием (т.е. архефолия).

12. Большая часть энергии и информации Вселенной скрыта в дырках пространственно-временного континуума. В них же скрыта от нас и темная материя. Поэтому, и общую, и специальную теорию относительности пора пересматривать. Точно также, причиной дуализма корпускула-волна, т.е. того, что микрочастица является одновременно и волной, и частицей, а также дуализма бытия-небытия и дуализма бифуркаций-фракталов является несплошность континуума и архефолия. Т.е., наличие дырок в физическом континууме является причиной дуализмов бытия-небытия, волнычастицы, бифуркации-фрактала, ничто-нечто ...

13. Предложенная нами теория и новая система и мироздания, и науки, дает также философское объяснение того, почему давно известные так называемые комплексные числа нельзя сравнивать друг с другом, т.е. нельзя спрашивать, какое из них больше, а какое меньше. Стало понятно, почему. Потому, что они разные не количественно, а качественно. Т.е. каждое комплексное «число» отличается и от других комплексных «чисел», и чисел вообще, качественно. Все они совершенно разные вещи в философском смысле, потому и не сравнимые. Т.е. любые качественно разные вещи - несравнимые, а любые несравнимые вещи - качественно разные.

14. $\quad$ У точки размер равен нулю, у дырки же размер больше нуля и есть границы. А вообще-то, если ширина вещи равна нулю, то длины у нее быть не может?

15. Пеано, сам того не подозревая, интуитивно пришел к своим пяти аксиомам конструктивного построения натурального ряда. Сам Пеано этого не знал, но в этих аксиомах совершенно незаметно, в скрытой форме выражена мысль, что, например, 5 может быть больше 3 только в том случае, если между 3 и 5 есть еще что-то. Вот это «что-то» и есть то связующее трех и пяти. Например, в данном примере это - число 4. И благодаря именно этому числу возникает и происходит философское отношение R. Вот это отношение и способствует передаче информации.

16. И материальная линия, и материальная плоскость, тоже несплошные - они прерывистые и состоят из материальных точек и дырок. Траектории элементарных частиц тоже должны быть прерывистыми?

17. В предлагаемой системе понятие непрерывной функции исчезает. Это происходит потому, что непрерывного аргумента $\boldsymbol{x}$ не бывает ( $\boldsymbol{x}$ всегда прерывистый). Т.е. если линия действительно существует, то она прерывистая (т.е. это - отточие); если 
плоскость действительно существует, то она дырявая. Если же их (т.е. линий и плоскостей) нет, т.е. они - только воображаемые, то все равно, они дырявые, в любом случае. Не существует такое трансцендентное «число», которое при возведении в какую-либо степень давало бы другое трансцендентное число. Да и точного значения трансцендентного числа тоже нет.

18. Если между физическими телами или философскими вещами нет ничего материального, то расстояния между ними не существует. Расстояние возможно только в том случае, если промежуток между этими вещами заполнен чем-то материальным или бытием. Если линия нулевой ширины сплошная, то она обладать длиной не может!? Длина возникнет только в случае, если линия прерывистая, т.е. если между точками наличествуют дырки!? Философская ошибка Евклида в том и заключается, что длины без ширины не бывает. Если ширины нет, то это - небытие. А небытие, наверное, не имеет длины. Поэтому расстояние между точками быть может, а длины между точками быть не может. Элементарные частицы могут обладать размером (диаметром) только в случае, если они несплошные (дырявые). Принятое в теории относительности релятивистское сжатие оказывается возможным благодаря наличию промежутков между точками всего сущего. Уменьшение расстояния между точками и приводит к сжатию. Вселенная потому и смогла в свое время сжаться до точки, которая затем взорвалась (Большой взрыв), что двигалась со скоростью света и состояла из пустот, дырок (если, конечно же, продолжать предполагать, что теория относительности - теория правильная). Т.е. Большой взрыв произошел из-за взаимодействия точек с дырками этой первой начальной «точки». Если Вселенная снова сожмется в «точку», то она все равно взорвется.

19. Если продолжать использовать привычную и принятую сегодня систему математики, т.е. то, что за точку пересечения осей координат выбран ноль, то в дырках находятся трансцендентные «числа». Если же выбрать оси координат непривычно странным образом, например, за начало координат выбрать не ноль, а, скажем, число $\boldsymbol{\pi}$ (трансцендентное «число»), то в дырках окажутся рациональные числа. Всего же вариантов расположения осей $\boldsymbol{x}$ и $\boldsymbol{y}$ по отношению к друг другу, а стало быть, пересечения всех прямых и линий вообще, четыре: 1) ось $\boldsymbol{y}$ проходит через точку на оси $\boldsymbol{x}$ и точка оси $\boldsymbol{y}$ совпадает с точкой оси $\boldsymbol{x} ; 2$ ) ось $\boldsymbol{y}$ проходит через дырку в оси $\boldsymbol{x}$ и точка оси $\boldsymbol{y}$ расположена в дырке оси $\boldsymbol{x} ; 3)$ ось $\boldsymbol{y}$ проходит через ось $\boldsymbol{x}$ так, что дырка в оси $\boldsymbol{x}$ совпадает с дыркой в оси $\boldsymbol{y}$; 4) ось $\boldsymbol{y}$ проходит через ось $\boldsymbol{x}$ так, что точка на оси $\boldsymbol{x}$ оказывается в дырке оси $\boldsymbol{y}$. Из наших философских рассуждений выше получилось, что на точках расположены рациональные числа, а в дырках иррациональные «числа» и трансцендентные «числа». Но для нормальных чисел места в дырках нет. Поэтому мы должны исключить варианты 2 и 4 как невозможные. Значит, реально может быть лишь два варианта 1 и 3.

\section{References}

1. Laue M. Istoriia fiziki. [History of Physics] M., gos. izd. tekh.-teor. literatury, 1956, p. 5. 
2. Vezirov H.N., Vezirov-Kengerli F.H. Problemy filosofskoi $i$ sotsiologicheskoi kategorii aktual'nosti [Problems of the philosophical and sociological category of relevance]. Zhurnal «Nauchnye trudy» Instituta filosofii NANA, №2, 2018, pp. 29 - 48.

3. E.Bekkenbakh, R.Bellman. Vvedenie v neravenstva [Introduction to inequalities]. M.: Mir, 1965, p.7.

4. Vezirov H.N., Vezirov-Kengerli F.H. Nekotorye problemy postsovetskikh respublik [Some problems of the post-Soviet republics]. Baku, "Ecoprint», 2018, $234 \mathrm{~s}$.

5. Evklid. Nachala [Beginnings]. T.1, M.-L., Giz.TTL, 1950, p. 11.

6. Pogorelov A.V. Geometriia. Uchebnik dlia 7-11 klassov obshcheobrazovatel'nykh uchrezhdenii [Geometry. Textbook for 7 - 11 grades of educational institutions]. M., «Prosveshchenie», 1998, pp. $6,65,74$.

7. Prigogine I., Stengers I. Poriadok iz khaosa [Order out of chaos]. M., «Progress», 1986, p. 371

8. Hegel G.W.F. Nauka logiki [Science of logic]. V.1, M., «Mysl'», 1970, p.140.

Translation of the Title, and References to the Author's Language

ФИЛОСОФСКО-МАТЕМАТИЧЕСКИЙ ПАРАДОКС ТОЧЕК

Хикмет Ниязи оглу Везиров, доктор физ.-мат. наук, руководитель лаборатории Института физики НАНА,

\section{Фикрет Хикмет оглу Везиров-Кенгерли}

E-mail: vezirov55@mail.ru

\section{Л И Т Е Р А Т У Р А}

1. М.Лауэ. История физики. М., гос. изд. тех.-теор. литературы, 1956, с. 5.

2. Х.Н.Везиров, Ф.Х.Везиров-Кенгерли. Проблемы философской и социологической категории актуальности. Журнал «Научные труды» Института философии НАНА, №2, 2018, c. $29-48$.

3. Э.Беккенбах, Р.Беллман. Введение в неравенства. М.: Мир, 1965, с.7.

4. Х.Н.Везиров, Ф.Х.Везиров-Кенгерли. Некоторые проблемы постсоветских республик. Баку, "Ecoprint», 2018, 234 c.

5. Евклид. Начала. Т.1, М.-Л., Гиз.ТТЛ, 1950, с. 11.

6. А.В.Погорелов. Геометрия. Учебник для 7 - 11 классов общеобразовательных учреждений. М., «Просвещение», 1998, с.с. 6, 65, 74.

7. И.Пригожин, И. Стенгерс. Порядок из хаоса. М., «Прогресс», 1986, с. 371.

8. Г.В.Ф.Гегель. Наука логики. Т.1, М., «Мысль», 1970, с.140. 